(C) The Authors 2016. This is an Open Access article, distributed under the terms of the Creative

Commons Attribution licence (http://creativecommons.org/licenses/by/4.0/), which permits unrestricted

re-use, distribution, and reproduction in any medium, provided the original work is properly cited.

\title{
The independent prospective associations of activity intensity and dietary energy density with adiposity in young adolescents
}

\author{
Esther M. F. van Sluijs ${ }^{1 *}$, Stephen J. Sharp ${ }^{1}$, Gina L. Ambrosini ${ }^{2,3}$, Aedin Cassidy $^{4}$, Simon J. Griffin ${ }^{1}$ and \\ Ulf Ekelund ${ }^{1,5}$ \\ ${ }^{1}$ School of Clinical Medicine, Medical Research Council Epidemiology Unit \& UK Clinical Research Collaboration (CRC) Centre \\ for Diet and Activity Research, University of Cambridge, Cambridge CB2 OQQ, UK \\ ${ }^{2}$ Medical Research Council Human Nutrition Research, Cambridge CB1 9NL, UK \\ ${ }^{3}$ School of Population Health, The University of Western Australia, Perth, WA 6009, Australia \\ ${ }^{4}$ Department of Nutrition, Norwich Medical School, University of East Anglia, Norwich NR4 7UQ, UK \\ ${ }^{5}$ Norwegian School of Sport Science, 0806 Oslo, Norway \\ (Submitted 1 June 2015 - Final revision received 20 October 2015 - Accepted 17 November 2015 - First published online 13 January 2016)
}

\section{Abstract}

There is limited evidence on the prospective association of time spent in activity intensity (sedentary (SED), moderate (MPA) or vigorous (VPA) physical activity) and dietary intake with adiposity indicators in young people. This study aimed to assess associations between (1) baseline objectively measured activity intensity, dietary energy density (DED) and 4-year change in adiposity and (2) 4-year change in activity intensity/DED and adiposity at follow-up. We conducted cohort analyses including 367 participants (10 years at baseline, 14 years at follow-up) with valid data for objectively measured activity (Actigraph), DED (4-d food diary), anthropometry (waist circumference (WC), \%body fat (\%BF), fat mass index (FMI), weight status) and covariates. Linear and logistic regression models were fit, including adjustment for DED and moderateto-vigorous physical activity. Results showed that baseline DED was associated with change in WC ( $\beta$ for $1 \mathrm{~kJ} / \mathrm{g}$ difference: $0 \cdot 71 ; 95 \% \mathrm{CI} 0 \cdot 26,1 \cdot 17)$, particularly in boys $(1 \cdot 26 ; 95 \%$ CI $0 \cdot 41,2 \cdot 16 v$. girls: $0 \cdot 26 ; 95 \%$ CI $-0 \cdot 34,0 \cdot 87)$, but not with \%BF, FMI or weight status. In contrast, baseline SED, MPA or VPA were not associated with any of the outcomes. Change in DED was negatively associated with FMI $(\beta$ for $1 \mathrm{~kJ} / \mathrm{g}$ increase: $-0 \cdot 86$; $95 \% \mathrm{CI}-1 \cdot 59,-0 \cdot 12)$ and $\% \mathrm{BF}(-0 \cdot 86 ; 95 \% \mathrm{CI}-1 \cdot 25,-0 \cdot 11)$ but not WC $(-0 \cdot 27 ; 95 \% \mathrm{CI}-1 \cdot 02,0 \cdot 48)$. Change in SED, MPA and VPA did not predict adiposity at follow-up. In conclusion, activity intensity was not prospectively associated with adiposity, whereas the directions of associations with DED were inconsistent. To inform public health efforts, future studies should continue to analyse longitudinal data to further understand the independent role of different energy-balance behaviours in changes in adiposity in early adolescence.

Key words: Physical activity: Sedentary behaviours: Dietary energy density: Adiposity: Prospective cohort studies: Epidemiology: Children and adolescents

The development of obesity is known to be largely the result of energy imbalance, in which energy intake (EI) exceeds energy expenditure over a prolonged period ${ }^{(1)}$. The most recent UK prevalence data show that when leaving primary school, one in three children are either overweight or obese ${ }^{(2)}$. A similar prevalence has been reported in the USA ${ }^{(3)}$, indicating a need for public health action. A recent meta-analysis of interventions for preventing obesity in children showed that those targeting changes in both physical activity and dietary behaviour were marginally more effective in reducing weight gain than those only targeting one ${ }^{(4)}$, although through what behavioural mechanism this is achieved is unclear. Although this observation is consistent with abundant cross-sectional evidence, the precise role of activity and diet behaviours in adiposity development remains unclear ${ }^{(5)}$. Moreover, the limited available evidence exhibits a number of limitations including the use of imprecise measures of activity, insufficient consideration of the impact of changes in the exposures and limited adjustment for confounding ${ }^{(6-8)}$.

Recent reviews highlight the lack of evidence of an association between objectively measured physical activity ${ }^{(8,9)}$ or sedentary time ${ }^{(6)}$ and changes in adiposity in young people. It has been suggested that the lack of association between total physical activity and subsequent changes in adiposity may mask associations with subcomponents of physical activity ${ }^{(9)}$. Indeed, a cross-sectional study suggests that activity of vigorous intensity is more strongly associated with adiposity outcomes than moderate activity or total physical activity ${ }^{(10)}$. One recent

Abbreviations: DED, dietary energy density; EI, energy intake; FMI, fat mass index; MVPA, moderate-to-vigorous physical activity; SED, sedentary physical activity; VPA, vigorous physical activity; WC, waist circumference.

* Corresponding author: Dr E. M. F. van Sluijs, fax +44 1223 330316, email esther.vansluijs@mrc-epid.cam.ac.uk 
prospective study additionally showed that, independent of diet quality and activity of lower intensity, participation in objectively measured vigorous activity at baseline was associated with a beneficial change in several health outcomes over a 2-year follow-up period ${ }^{(11)}$. However, this study only considered a limited number of confounders (age, sex and diet quality assessed with 24-h recall) and did not assess the impact of change in behavioural exposures. Moreover, despite suggestions that time spent sedentary may be an important independent risk factor for children's health ${ }^{(12,13)}$, recent prospective studies have failed to confirm this association ${ }^{(14,15)}$.

In contrast, the limited available prospective epidemiological evidence consistently indicates energy-dense diets as a contributing factor to EI and excess weight gain in childhood ${ }^{(7,16,17)}$. Dietary energy density (DED) refers to the amount of energy consumed given the weight of food reportedly consumed, and therefore likely to be less prone to under-reporting than the absolute measure of EI. Prospectively, DED has been associated with subsequent changes in adiposity in childhood, independent of $\mathrm{EI}^{(18)}$. However, no study has investigated changes in DED and adiposity, and few prospective studies adjust for the potential contribution of physical activity ${ }^{(19)}$. It therefore remains unclear whether and how physical activity and dietary factors influence weight development in children.

In light of the limitations of the current evidence base, we aimed to quantify the independent association between (change in) activity intensity (e.g. time spent in sedentary, moderate or vigorous activity) and DED and change in adiposity over a 4-year follow-up period in a population-based sample of young British adolescents. We tested the following complementary hypotheses: (1) behaviour at baseline predicts change in adiposity; and (2) change in behaviour predicts adiposity at follow-up.

\section{Methods}

\section{Study overview}

The SPEEDY study (Sport, Physical activity and Eating behavior: Environmental Determinants in Young people) is a populationbased longitudinal cohort study set in the county of Norfolk, $\mathrm{UK}^{(20)}$. Ethics approval for the whole study was obtained from the University of East Anglia Research Ethics Committee; parental informed consent and student assent were obtained at all measurement occasions. The analyses presented here used data from baseline and 4-year follow-up.

\section{Study procedures}

The complete details of SPEEDY participant recruitment and study procedures for the baseline ${ }^{(20)}$ and follow-up ${ }^{(21)}$ data collection have been detailed elsewhere. In brief, at baseline, primary schools in Norfolk were purposively sampled to achieve urban and rural heterogeneity. In total, 157 schools were approached (out of 227 eligible schools with $\geq 12$ Year 5 children), of which ninety-two were recruited and participated in the study. Invitation packs were handed out to all Year 5 children ( $n$ 3619, aged 9-10 years). A total of 2064 children provided valid consent and were measured at baseline ( $57 \%$ response rate). Baseline data collection took place during school visits between April and July 2007. At 4-year follow-up, all the participants with valid baseline home addresses ( $n$ 1964) were sent an invitation pack. Researchers additionally gave presentations at secondary schools attended by at least five original SPEEDY participants to encourage participation. Consent forms (signed by both parents and participants) were returned to the study office by mail. Follow-up data collection took place at schools (or at home if more convenient) between April and August 2011.

\section{Data collection procedures}

At both time points, researchers visited schools to take physical measurements, administer self-report questionnaires, fit accelerometers and hand out 4-d food diaries; participants returned the accelerometers and the diaries to school 1 week later.

Anthropometry assessment. Trained research assistants used standardised protocols to measure participants' height and weight. Height was measured to the nearest millimetre (Leicester height measure; Chasmors Ltd). A non-segmental bio-impedance scale was used to measure weight (to the nearest $0.1 \mathrm{~kg}$ ) and impedance in light clothing (type TBF-300A; Tanita). Height and weight measures were used to calculate BMI $\left(\mathrm{kg} / \mathrm{m}^{2}\right)$. Weight status was derived using International Obesity Task Force sex- and age-dependent cut-off points ${ }^{(22)}$. Previously validated and published procedures ${ }^{(23)}$ using eight equations were used to calculate fat mass and body fat percentage ${ }^{(24-31)}$. Fat mass index (FMI) was presented as fat mass $/$ height $^{2}\left(\mathrm{~kg} / \mathrm{m}^{2}\right)$. Waist circumference (WC) was measured twice to the nearest millimetre at the midpoint between the lower costal margin and the level of the anterior superior iliac crests, using a Seca 200 measuring tape (Seca). A third measurement was taken if a discrepancy of $\geq 3 \mathrm{~cm}$ was observed and an average was calculated. All scales were calibrated before and halfway through data collection. Quality assurance was established by assessing inter-observer variability of height and WC before and after data collection, which was found to be acceptable (ranging from 0.1 to $0.4 \mathrm{~cm}$ for height and 0.7 to $1.3 \mathrm{~cm}$ for WC).

Physical activity assessment. Physical activity was objectively assessed using an Actigraph accelerometer (model GT1M; Actigraph). The Actigraph has been shown to have validity in assessing physical activity among children during free-living conditions ${ }^{(32,33)}$, although it cannot accurately assess waterbased activities and cycling. All monitors were calibrated before first use and regularly throughout the study. The monitor was set to record the vertical acceleration at 5-s epochs. Participants were asked to wear the monitors during waking hours for $7 \mathrm{~d}$ and to remove them while undertaking water-based activities.

Data were analysed using a batch processing programme (MAHUffe, available at: www.mrc-epid.cam.ac.uk/research/ resources) to remove any data recorded after 23.00 hours and before 06.00 hours. Periods of $10 \mathrm{~min}$ or more that had continuous zero activity counts and any days with $<500 \mathrm{~min}$ of recording were excluded ${ }^{(34,35)}$. Participants were included if they provided $\geq 3 \mathrm{~d}$ of valid data at both time points. 
Physical activity at baseline and at follow-up for each individual was summarised as average daily time spent sedentary (SED) and in moderate (MPA), vigorous (VPA) and moderateto-vigorous physical activity (MVPA). Thresholds for defining activity intensities were as follows: SED $<100$ counts per min (cpm), MPA 2000-3999 and VPA $\geq 4000 \mathrm{cpm}$; MVPA was defined as $\geq 2000 \mathrm{cpm}^{(36,37)}$ and scaled to 5 -s epochs.

Dietary assessment and processing. Dietary intake was assessed using a 4-d food diary - a method previously validated in 9-10-year-old children ${ }^{(38)}$ and applied in young adolescents ${ }^{(39)}$. Assessment days were consecutive, concurrent with physical activity measurement and included 2 weekdays and 2 weekend days. Participants recorded, with assistance of their parents or care givers, all foods and drinks consumed, and estimated the portion size of each item. Participants practiced completion during the measurement session by reporting on the foods and drinks consumed that day, on which they were given feedback to improve the level of detail provided. The weights of the portions were then approximated using published values for children ${ }^{(40-42)}$. Diaries were coded at Medical Research Council Human Nutrition Research (Cambridge, UK) using Diet-In Nutrients-Out ${ }^{(43)}$, which uses continually updated British food composition data.

Daily DED $(\mathrm{kJ} / \mathrm{g})$ was estimated as total EI from food $(\mathrm{kJ})$ relative to total grams of food consumed, excluding beverages and supplements, which has been shown to provide a more valid estimate of $\mathrm{DED}^{(44)}$. Beverages included the following: tea, coffee, other hot beverages, water (drink), milk (drink), milkshakes, dairy smoothies (excluding fruit smoothies), yogurt drinks, diluted juices/squash, fruit juices and all carbonated and non-carbonated soft drinks. Items added to beverages, including sugar, dried beverage powders and milk were excluded from DED calculations.

Dietary misreporting can bias diet-disease associations, and dietary under-reporting is common among adolescents ${ }^{(45)}$. We quantified dietary misreporting as the ratio of daily EI relative: estimated energy requirements (EER, estimated as total energy expenditure plus energy required for growth) ${ }^{(46)}$. As cut-offs to identify under- and over-reporters may be subject to error ${ }^{(47)}$ and as the average EI:EER at baseline was 0.67 (SD 0.16), indicating that under-reporting was very common in this cohort, EI:EER was included as a continuous variable ${ }^{(48)}$.

Covariates. Inclusion of covariates focused on accounting for alternative important determinants of adiposity in youth, such as parental weight status, birth weight, sleep duration and puberty status ${ }^{(49-51)}$. Data on maternal BMI (calculated from self-reported height and weight), parent-reported child's birth weight and age when the main care giver left full-time education (self-reported and categorised as $\leq 16,16-18$, $>18$ years) were collected via a self-administered parental baseline questionnaire. At the age of 14 years, parents reported on five puberty signs from which a sum-score (0-5) was derived - three general (growth spurt, body hair growth, spots/ acne) and two sex-specific (boys: deepening voice, facial hair; girls: breast growth, menarche) scores. Average sleep duration at baseline was derived from the child's self-reported bedtime and wake time during school days and weekends, with the mean recorded as average sleep duration in hours ${ }^{(10)}$. Age and sex were self-reported during the measurement sessions; average accelerometer wear time $(\mathrm{min} / \mathrm{d})$ was derived from the processed accelerometer data; and DED from drinks and dietary misreporting were established using the procedures described above.

\section{Statistical analyses}

All the analyses were performed using STATA version 13 . Baseline age, sex, BMI $Z$ score and the care giver's age when leaving full-time education were compared between those included and excluded from the analysis, with $P$ values for these comparisons calculated from logistic regression with exclusion/inclusion as the binary outcome, and robust standard errors to allow for school-level clustering. We then modelled four adiposity variables (WC, FMI, \%body fat (\%BF) and weight status (overweight/obese $v$. normal weight)) and four exposure variables (time spent in SED, MPA and VPA and DED). For each outcome/exposure combination, a series of linear (continuous outcome) or logistic (binary outcome) regression models were fit, with robust standard errors to account for clustering within schools. Two complementary analytical approaches were used for each outcome/exposure combination:

(A) Regression of outcome on baseline exposure, adjusted for baseline value of outcome (e.g. baseline SED with change in FMI).

(B) Regression of outcome on change in exposure (e.g. change in SED with follow-up FMI).

First, a basic model with adjustment for age and sex was run for all analyses (model 1). Subsequent models also included socio-economic status, birth weight, maternal BMI, puberty status at follow-up and sleep (model 2); reciprocal adjustment for MVPA and DED (model 3); and reciprocal adjustment for MVPA and SED (activity-related exposures only, model 4). All models with activity-related exposures were additionally adjusted for baseline/change in accelerometer wear time; DED models were additionally adjusted for baseline/change in DED from drinks ${ }^{(44)}$ and baseline under-reporting. Models with WC as the outcome were adjusted for height at baseline. To account for the potential association between baseline behaviour and change in behaviour, all models including change in behaviour were additionally adjusted for the baseline value of that behaviour. Differences in exposure/outcome associations by sex and baseline weight status were investigated by including an interaction term between the moderating variable and the main exposure of interest in model 3. Effects in subgroups were estimated when the $P$ value for the test of interaction was $<0 \cdot 1$. To assess the influence of missing data, we ran sensitivity analyses restricting the sample to those with full data only. Differences from the main results were minimal and did not affect conclusions (data not reported).

\section{Results}

Of 2064 baseline participants, 1964 (95.2) had valid contact details, of which 480 were re-recruited at the 4-year follow-up 
Table 1. Baseline characteristics and observed change in exposures and outcomes in the analytical sample (Mean values and standard deviations)

\begin{tabular}{|c|c|c|c|c|}
\hline & \multicolumn{2}{|c|}{$\begin{array}{l}\text { Boys } \\
(n \text { 175) }\end{array}$} & \multicolumn{2}{|c|}{$\begin{array}{c}\text { Girls } \\
(n 192)\end{array}$} \\
\hline & Mean/\% & $\mathrm{sD} / n$ & Mean/\% & $\mathrm{sD} / n$ \\
\hline \multicolumn{5}{|l|}{ Demographic data } \\
\hline Age (years) & $9 \cdot 7$ & 0.5 & 9.8 & 0.4 \\
\hline Maternal BMI (kg/m²) & $24 \cdot 8$ & 4.9 & $25 \cdot 0$ & 4.7 \\
\hline Sleep time $(h)$ & $10 \cdot 4$ & 0.9 & $10 \cdot 7$ & 0.7 \\
\hline \multicolumn{5}{|c|}{ Age the care giver left full-time education (\%) } \\
\hline$\leq 16$ years & 33.7 & 59 & 43.8 & 84 \\
\hline $16-18$ years & 38.9 & 68 & 34.4 & 66 \\
\hline$>18$ years & $22 \cdot 9$ & 40 & $20 \cdot 3$ & 39 \\
\hline \multicolumn{5}{|l|}{ Anthropometry } \\
\hline \multicolumn{5}{|l|}{ Waist (cm) } \\
\hline Baseline & $64 \cdot 3$ & $7 \cdot 6$ & $62 \cdot 9$ & $7 \cdot 8$ \\
\hline 4-year change & $8 \cdot 2$ & $5 \cdot 2$ & 8.8 & $5 \cdot 6$ \\
\hline \multicolumn{5}{|l|}{ FMI $\left(\mathrm{kg} / \mathrm{m}^{2}\right)$} \\
\hline Baseline & 7.9 & 3.8 & 9.4 & 4.9 \\
\hline 4-year change & 4.4 & 4.2 & $7 \cdot 8$ & 4.5 \\
\hline \multicolumn{5}{|l|}{ \%body fat } \\
\hline Baseline & $21 \cdot 0$ & $5 \cdot 6$ & 24.9 & $6 \cdot 1$ \\
\hline 4-year change & -0.5 & 3.9 & 4.5 & 4.1 \\
\hline \%overweight/obese (baseline) & 18.5 & 32 & 23.4 & 45 \\
\hline \multicolumn{5}{|l|}{ Diet diary-derived data } \\
\hline \multicolumn{5}{|l|}{ DED (kJ/g) } \\
\hline Baseline & $7 \cdot 6$ & 1.3 & 7.9 & 1.3 \\
\hline 4-year change & 0.6 & 1.5 & 0.3 & 1.6 \\
\hline \multicolumn{5}{|l|}{ Accelerometer-derived data } \\
\hline \multicolumn{5}{|l|}{ SED (min) } \\
\hline Baseline & 453.4 & $51 \cdot 3$ & 468.0 & $52 \cdot 8$ \\
\hline 4-year change & $48 \cdot 0$ & $72 \cdot 6$ & $41 \cdot 1$ & 65.8 \\
\hline \multicolumn{5}{|l|}{ MPA (min) } \\
\hline Baseline & $53 \cdot 6$ & $13 \cdot 9$ & $45 \cdot 2$ & $11 \cdot 8$ \\
\hline 4-year change & -9.9 & $17 \cdot 8$ & $-1 \cdot 6$ & $16 \cdot 6$ \\
\hline \multicolumn{5}{|l|}{ VPA (min) } \\
\hline Baseline & $30 \cdot 9$ & $15 \cdot 6$ & $22 \cdot 0$ & $11 \cdot 1$ \\
\hline 4-year change & -7.4 & $17 \cdot 0$ & $-6 \cdot 4$ & $15 \cdot 4$ \\
\hline \multicolumn{5}{|l|}{ Wear time (min) } \\
\hline Baseline & $722 \cdot 8$ & $55 \cdot 5$ & $715 \cdot 7$ & $61 \cdot 2$ \\
\hline 4-year follow-up & $716 \cdot 0$ & $70 \cdot 8$ & $703 \cdot 8$ & $61 \cdot 2$ \\
\hline
\end{tabular}

(24.4\%); 424 (88\%) returned an Actigraph monitor, with 367 (76.4\% of follow-up sample) providing valid data at both baseline and follow-up on physical activity and/or DED. Those included in the analyses ( $n$ 367) did not differ from those excluded ( $n$ 1697) with respect to sex and baseline age and BMI $Z$ scores. However, on average, they did come from more highly educated families (age parent left full-time education ( $\leq 16, \quad 16-18,>18$ years): included: 30.0, 36.5, $21.5 \% v$. excluded: $44.9,27 \cdot 9,17 \cdot 4 \% ; P=0.027)$. Table 1 shows baseline characteristics and mean change in exposure, outcome and confounding variables, stratified by sex.

Table 2 shows the results of linear regression analyses using baseline exposure and change in adiposity (analytical approach A). The activity-related exposures were not associated with change in any of the outcomes. Baseline DED was, however, positively associated with change in WC independent of baseline MVPA. Only one significant interaction was identified, suggesting that the main effect of baseline DED on change in WC differed by sex. Subsequent subgroup analyses indicated that the positive effect was statistically significant in boys but not in girls ( $\beta$ for boys: $1 \cdot 28$; $95 \% \mathrm{CI} 0 \cdot 41,2 \cdot 16 v$. girls: $0 \cdot 26$; $95 \% \mathrm{CI}-0 \cdot 34,0 \cdot 87$ ).
Table 3 presents the results of linear regression analyses of change in exposure and adiposity at follow-up (analytical approach B). For the activity-related exposures, the estimated associations were generally not in the expected direction, although the $95 \%$ CI were wide and compatible with no association. In contrast, after adjustment for confounders (model 2), change in DED was significantly, and negatively, associated with FMI and \%BF at follow-up, but not with WC. Further adjustment for MVPA made very little difference to the estimates of association. Only one significant interaction was identified, suggesting that the effect of changes in VPA on FMI at follow-up differed by obesity status. However, although the estimated $\beta$-coefficients were in opposite directions in normal weight and overweight/obese children, both $95 \%$ CI were compatible with no association ( $\beta$ for normal weight: $0 \cdot 46$; $95 \%$ CI $-0 \cdot 18$, 1.11, overweight/obese: $-1 \cdot 13$; $95 \%$ CI $-3 \cdot 92,0 \cdot 17)$.

The results of logistic regression analyses using weight status as the outcome are presented in Table 4. There was very little evidence to suggest that either baseline behaviour or change in behaviour was associated with being overweight/obese at follow-up. Increasing SED was associated with lower odds of 
Table 2. Associations between baseline behaviour and change in adiposity (analytical approach $A)^{*}$ ( $\beta$-Coefficients and $95 \%$ confidence intervals)

\begin{tabular}{|c|c|c|c|c|c|c|}
\hline \multirow[b]{2}{*}{ Behaviours } & \multicolumn{2}{|c|}{ WC } & \multicolumn{2}{|c|}{ FMI } & \multicolumn{2}{|c|}{$\%$ body fat } \\
\hline & $\beta$ & $95 \% \mathrm{Cl}$ & $\beta$ & $95 \% \mathrm{Cl}$ & $\beta$ & $95 \% \mathrm{Cl}$ \\
\hline \multicolumn{7}{|l|}{$\operatorname{SED}(\min / \mathrm{d}) \dagger$} \\
\hline Model $1 \neq$ & -0.14 & $-0.32,0.04$ & -0.02 & $-0.17,0.13$ & 0.02 & $-0.09,0.14$ \\
\hline Model $2 \S$ & -0.12 & $-0.31,0.07$ & -0.01 & $-0.16,0.14$ & 0.01 & $-0.11,0.13$ \\
\hline Model 3॥ & -0.14 & $-0.31,0.03$ & -0.06 & $-0.21,0.08$ & -0.04 & $-0.13,0.05$ \\
\hline Model 4ף & -0.10 & $-0.43,0.23$ & -0.11 & $-0.40,0.19$ & -0.05 & $-0.25,0.14$ \\
\hline \multicolumn{7}{|l|}{ MPA $(\min / \mathrm{d}) \dagger$} \\
\hline Model $1 \neq$ & 0.49 & $0.00,0.99$ & 0.05 & $-0.27,0.38$ & -0.05 & $-0.35,0.25$ \\
\hline Model $2 \S$ & 0.54 & $-0.01,1 \cdot 10$ & 0.06 & $-0.25,0.38$ & -0.03 & $-0.32,0.27$ \\
\hline Model 3॥ & 0.57 & $-0.01,1 \cdot 15$ & 0.20 & $-0.17,0.58$ & 0.11 & $-0.19,0.40$ \\
\hline Model 4ף & 0.54 & $-0.45,1.53$ & 0.11 & $-0.56,0.78$ & 0.003 & $-0.59,0.60$ \\
\hline \multicolumn{7}{|l|}{ VPA $(\min / \mathrm{d}) \dagger$} \\
\hline Model $1 \neq$ & 0.22 & $-0.24,0.69$ & -0.05 & $-0.40,0.29$ & -0.09 & $-0.39,0.20$ \\
\hline Model $2 \S$ & 0.06 & $-0.37,0.49$ & -0.18 & $-0.44,0.08$ & -0.15 & $-0.43,0.13$ \\
\hline Model 3॥ & 0.20 & $-0.22,0.62$ & -0.01 & $-0.25,0.23$ & 0.05 & $-0.19,0.28$ \\
\hline Model 4ף & -0.09 & $-0.71,0.52$ & -0.20 & $-0.65,0.26$ & -0.04 & $-0.39,0.31$ \\
\hline \multicolumn{7}{|l|}{$\mathrm{DED}^{* *}$} \\
\hline Model $1 \ddagger$ & 0.67 & $0.25,1.09$ & 0.31 & $0.07,0.55$ & 0.27 & $0.03,0.52$ \\
\hline Model $2 \S$ & 0.69 & $0.23,1.15$ & 0.22 & $-0.08,0.52$ & 0.18 & $-0.14,0.50$ \\
\hline Model 3॥ & 0.71 & $0.26,1.17$ & 0.22 & $-0.08,0.52$ & 0.18 & $-0.14,0.50$ \\
\hline
\end{tabular}

WC, waist circumference; FMI, fat mass index; SED, sedentary physical activity; MPA, moderate physical activity; VPA, vigorous physical activity; DED, dietary energy density; PA, physical activity; MPVA, moderate-to-vigorous physical activity.

* $\beta$ Represents the estimated difference in mean change in outcome per $10 \mathrm{~min}$ (PA/SED) or $1 \mathrm{~kJ} / \mathrm{g}$ (DED) increase in exposure. $n$ included in the analyses varies between 245 and 336, depending on exposure and outcome.

$\uparrow$ Additionally adjusted accelerometer-registered time.

$\ddagger$ Model 1: age, sex.

$\S$ Model 2: model 1 + socio-economic status, birth weight, maternal BMI, puberty status at follow-up, sleep duration; model with WC as outcome also adjusted for height.

II Model 3: model 2 + baseline DED for PA exposures and baseline MVPA for DED.

I Model 4: model 3+objectively measured sedentary time (for MPA and VPA exposures) or MVPA (for SED exposure).

** DED models additionally adjusted for energy intake $(\mathrm{kJ})$ from drinks and baseline under-reporting.

Table 3. Associations between change in behaviour and adiposity at follow-up (analytical approach B) ${ }^{\star}$ ( $\beta$-Coefficients and $95 \%$ confidence intervals)

\begin{tabular}{|c|c|c|c|c|c|c|}
\hline & \multicolumn{2}{|c|}{ WC } & \multicolumn{2}{|c|}{ FMI } & \multicolumn{2}{|c|}{ \%body fat } \\
\hline & $\beta$ & $95 \% \mathrm{Cl}$ & $\beta$ & $95 \% \mathrm{Cl}$ & $\beta$ & $95 \% \mathrm{Cl}$ \\
\hline \multicolumn{7}{|l|}{$\operatorname{SED}(\min / \mathrm{d}) \dagger$} \\
\hline Model $1 \ddagger$ & -0.03 & $-0.30,0.23$ & -0.04 & $-0.24,0.15$ & -0.07 & $-0.23,0.10$ \\
\hline Model $2 \S$ & -0.01 & $-0.24,0.23$ & -0.06 & $-0.24,0.12$ & -0.05 & $-0.22,0.11$ \\
\hline Model 3॥ & -0.002 & $-0.27,0.27$ & -0.09 & $-0.30,0.12$ & -0.09 & $-0.27,0.08$ \\
\hline Model 4ף & 0.24 & $-0.25,0.72$ & 0.17 & $-0.23,0.57$ & 0.09 & $-0.23,0.40$ \\
\hline \multicolumn{7}{|l|}{ MPA $(\min / d) \dagger$} \\
\hline Model $1 \ddagger$ & 0.37 & $-0.31,1.05$ & 0.19 & $-0.34,0.71$ & 0.25 & $-0.23,0.72$ \\
\hline Model $2 \S$ & 0.16 & $-0.50,0.81$ & 0.08 & $-0.46,0.62$ & 0.12 & $-0.38,0.63$ \\
\hline Model 3॥ & 0.10 & $-0.63,0.83$ & 0.02 & $-0.58,0.62$ & 0.03 & $-0.47,0.54$ \\
\hline Model 4ף & 0.03 & $-1.04,1.11$ & -0.10 & $-1.00,0.81$ & -0.23 & $-0.91,0.44$ \\
\hline \multicolumn{7}{|l|}{ VPA $(\min / d) \dagger$} \\
\hline Model $1 \ddagger$ & 0.35 & $-0.33,1.02$ & 0.24 & $-0.33,0.80$ & 0.26 & $-0.24,0.76$ \\
\hline Model $2 \S$ & 0.36 & $-0.43,1.14$ & 0.19 & $-0.45,0.84$ & 0.19 & $-0.37,0.76$ \\
\hline Model 3॥ & 0.30 & $-0.58,1.18$ & 0.39 & $-0.30,1.09$ & 0.40 & $-0.22,1.02$ \\
\hline Model 4ף & 0.48 & $-0.49,1.45$ & 0.51 & $-0.26,1.28$ & 0.47 & $-0.22,1.15$ \\
\hline \multicolumn{7}{|l|}{ DED ${ }^{\star *}$} \\
\hline Model 1‡ & -0.43 & $-1 \cdot 20,0.34$ & -0.63 & $-1.23,-0.02$ & -0.52 & $-1.03,-0.01$ \\
\hline Model $2 \S$ & -0.26 & $-1.01,0.49$ & -0.83 & $-1.56,-0.10$ & -0.66 & $-1.22,-0.09$ \\
\hline Model 3॥ & -0.27 & $-1.02,0.48$ & -0.86 & $-1.59,-0.12$ & -0.68 & $-1 \cdot 25,-0.11$ \\
\hline
\end{tabular}

WC, waist circumference; FMI, fat mass index; SED, sedentary physical activity; MPA, moderate physical activity; VPA, vigorous physical activity; DED, dietary energy density; PA, physical activity; MPVA, moderate-to-vigorous physical activity.

* $\beta$ Represents the estimated difference in mean level of outcome per $10 \mathrm{~min}$ (PA/SED) or $1 \mathrm{~kJ} / \mathrm{g}$ (DED) increase in exposure. $n$ included in the analyses varies between 245 and 335 , depending on exposure and outcome.

$\dagger$ Additionally adjusted for change in accelerometer-registered time.

† Model 1: age, sex, baseline value of exposure.

$\S$ Model 2: model 1 + socio-economic status, birth weight, maternal BMI, puberty status at follow-up, sleep duration; model with WC as outcome also includes height.

II Model 3: model 2 + change in DED for PA/SED exposures and change in MVPA for DED.

Tl Model 4: model 3+objectively measured change in sedentary time (for MPA and VPA exposures) or change in MVPA (for SED exposure).

** DED models additionally adjusted for change energy intake $(\mathrm{kJ})$ from drinks and baseline under-reporting. 
Table 4. Associations between baseline behaviour/change in behaviour and odds of being overweight/obese at follow-up* (Odds ratios and $95 \%$ confidence intervals)

\begin{tabular}{|c|c|c|c|c|}
\hline & \multicolumn{2}{|c|}{ Baseline behaviourt } & \multicolumn{2}{|c|}{ Change in behaviour } \\
\hline & OR & $95 \% \mathrm{Cl}$ & OR & $95 \% \mathrm{Cl}$ \\
\hline \multicolumn{5}{|l|}{$\operatorname{SED}(\mathrm{min} / \mathrm{d}) \ddagger$} \\
\hline Model $1 \S$ & 1.07 & $0.97,1.18$ & 0.94 & $0.88,1.01$ \\
\hline Model 2\| & 1.05 & $0.96,1.15$ & 0.92 & $0.84,1.00$ \\
\hline Model 39 & 1.01 & $0.91,1.13$ & 0.90 & $0.81,0.99$ \\
\hline Model $4^{\star *}$ & 1.02 & $0.85,1.22$ & 0.95 & $0.78,1.14$ \\
\hline \multicolumn{5}{|l|}{ MPA $(\min / \mathrm{d}) \ddagger$} \\
\hline Model $1 \S$ & 0.81 & $0.58,1.11$ & $1 \cdot 11$ & $0.94,1.31$ \\
\hline Model 2\| & 0.89 & $0.65,1.21$ & 1.09 & $0.89,1.33$ \\
\hline Model 39 & 1.00 & $0.69,1.46$ & $1 \cdot 11$ & $0.86,1.41$ \\
\hline Model $4^{\star \star}$ & $1 \cdot 13$ & $0.54,2.37$ & 0.92 & $0.65,1.29$ \\
\hline \multicolumn{5}{|l|}{ VPA $(\min / \mathrm{d}) \ddagger$} \\
\hline Model $1 \S$ & 0.73 & $0.53,1.00$ & $1 \cdot 13$ & $0.93,1.37$ \\
\hline Model 2\| & 0.78 & $0.53,1.16$ & $1 \cdot 13$ & $0.84,1.51$ \\
\hline Model 39 & 0.94 & $0.63,1.41$ & $1 \cdot 17$ & $0.87,1.57$ \\
\hline Model $4^{\star *}$ & 0.95 & $0.57,1.59$ & $1 \cdot 11$ & $0.77,1.60$ \\
\hline \multicolumn{5}{|l|}{ DED†† } \\
\hline Model $1 \S$ & $1 \cdot 18$ & $0.89,1.57$ & 1.02 & $0.83,1.26$ \\
\hline Model 2\| & 1.03 & $0.73,1.45$ & 0.94 & $0.71,1.23$ \\
\hline Model $3^{\star *}$ & 1.01 & $0.72,1.42$ & 0.94 & $0.72,1.22$ \\
\hline
\end{tabular}

SED, sedentary physical activity; MPA, moderate physical activity; VPA, vigorous physical activity; DED, dietary energy density; PA, physical activity; MVPA, moderate-to-vigorous physical activity.

* OR of being overweight/obese per $10 \mathrm{~min}$ (PA) or $1 \mathrm{~kJ} / \mathrm{g}$ (DED) increase in exposure. $n$ included in the analyses varies between 245 and 334, depending on exposure and outcome.

† Additional adjustment for BMI $Z$ score at baseline.

$\ddagger$ Additionally adjusted accelerometer-registered time.

$\S$ Model 1: age, sex.

II Model 2: model 1+socio-economic status, birth weight, maternal BMI, puberty status at follow-up, sleep duration.

Il Model 3: model 2 + baseline DED for PA exposures and baseline MVPA for DED.

** Model 4: model 3 +objectively measured sedentary time (for MPA and VPA exposures) or MVPA (for SED exposure).

†† DED models additionally adjusted for energy intake $(\mathrm{kJ})$ from drinks and baseline under-reporting.

overweight/obesity at follow-up; however, this association became non-significant after adjustment for change in MVPA. A significant interaction suggested that the influence of baseline SED on change in obesity status differed by sex. However, although the estimated OR were in opposite directions in boys and girls, both $95 \% \mathrm{CI}$ were compatible with no association (OR for girls: 0.97 ; $95 \%$ CI 0.82, 1.14, and boys: 1.25 ; $95 \%$ CI 0.92, 1.72).

\section{Discussion}

This study shows that, in early adolescence, objectively measured activity intensity is not prospectively associated with adiposity markers, whereas the direction of results for DED was inconsistent. We are therefore unable to draw robust conclusions about the importance of energy-balance behaviours for obesity prevention. Strengths of the study include the longitudinal design, the relatively large population-based sample with objectively measured physical activity, detailed food diary data and objective anthropometry measures at two time points in a challenging age group. However, a key limitation is the cohort attrition, impacting on the wider generalisability of the results, particularly as those included in the present analyses on average came from higher educated families than the original cohort.
As suggested by previous review evidence ${ }^{(7,16)}$, DED was associated with changes in indicators of adiposity. The results showed that a higher DED at baseline was associated with a greater 4-year increase in WC, but not FMI, \%BF or weight status. A higher baseline DED of $1 \mathrm{~kJ} / \mathrm{g}$ was associated with a $0.71 \mathrm{~cm}$ greater increase in WC. This association was independent of a number of potential confounders previously underexplored in the literature, such as birth weight, maternal BMI and sleep duration. Models were additionally adjusted for EI from drinks and dietary under-reporting; further adjustment for objectively measured physical activity did not attenuate the observed association. In addition to addressing the more commonly studied predictors of change in adiposity, we also investigated the association between changes in DED and adiposity indicators at follow-up. To our knowledge, no study has explored this in an adolescent population. Unexpectedly, the results showed that an increase in DED was associated with lower FMI and lower \%BF at follow-up, whereas no association was observed with WC. There are several hypotheses for the observed negative association. First, DED at baseline might have reached a ceiling level for children with high baseline adiposity, whereas increases in DED were feasible for smaller children at initially lower levels. Baseline weight status did not, however, moderate the association, raising doubt about this hypothesis. Second, changes in DED might have been due to changes in energy expenditure, and therefore changes in energy requirements. However, adjustment for minutes of MVPA did not attenuate the association. Third, reporting bias in dietary assessment is known to be weight dependent ${ }^{(45)}$. Analyses were adjusted for baseline dietary under-reporting, resulting in minimal attenuation (data not shown). The likely impact of potential changes in reporting bias due to increased weight is therefore considered minimal. Whatever the reason, the overall mixed results observed in this study prevent drawing robust conclusions about the longitudinal association between DED and adiposity in early adolescence and warrant further exploration in sufficiently large samples with multiple robust measures of exposure, outcome and potentially confounding variables.

The role of physical activity in weight gain has been a longstanding issue of discussion. Despite an abundance of evidence for a cross-sectional association, particularly for activity of higher intensity, overall, the results of longitudinal and interventional studies have been mixed ${ }^{(6,9,52)}$. In the present study, the results were consistent between activity-related exposures and generally counter-intuitive, but not statistically significant. Although one might argue that this may be due to a small sample size, the size of the effect estimates additionally indicates that the observed associations are unlikely to be of clinical relevance. Results from the logistic regression model using weight status at follow-up as the outcome showed that a 10-min increase in SED was associated with $10 \%$ lower odds of being overweight or obese at follow-up, after controlling for known confounders and DED. However, this association was attenuated and became non-significant after adjustment for MVPA. A recent review of longitudinal evidence on the association between objectively measured sedentary time and $\operatorname{adiposity}^{(6)}$ identified only three studies, two of which reported 
a null association with the remaining study showing a positive association. Longitudinal evidence that sedentary time, or indeed specific sedentary behaviours (in particular television viewing $)^{(53)}$, is positively associated with adiposity therefore remains weak at best. Although reductions in specific sedentary behaviours may be beneficial, calls to reduce overall sedentary time to reduce obesity at a population level may therefore be premature.

The common approach to modelling the prospective association between health behaviours and outcome is to regress change in outcome on baseline behaviour (as presented here in Table 2). The data presented here would have enabled development of a change model (where change in behaviour is related to change in adiposity). However, as in cross-sectional analyses, identification of the direction of association is not possible in these models, and this analytical strategy was therefore not pursued. The clear temporal sequences of the models presented in this study were hypothesised to provide a clearer indication of the potential direction of association, and therefore a step closer to the identification of any causal associations. However, the lack of associations for the activityrelated models and the conflicting results for the DED models prevent this.

Reverse causality in the obesogenic behaviour-adiposity relationship needs to be considered in light of the results presented in this study. Recent data in children suggest that adiposity predicts lower levels of physical activity ${ }^{(54)}$ and higher amounts of sedentary time ${ }^{(55)}$. This has also been suggested in adults $^{(56,57)}$. Although plausible, there are also methodological reasons for this observation. Both outcome and exposure variables are measured with error, but behavioural variables are generally measured with greater error than anthropometric data. Random measurement error in the exposure variable leads to an attenuation of the association to zero. However, random measurement error in the outcome variable increases the standard errors, and therefore impacts the precision, but not the estimate of effect ${ }^{(58)}$. Efforts to improve the validity and reduce measurement error of the measures of behaviour are therefore crucial to improve our understanding of the causality of the association under investigation.

\section{Conclusions}

This is one of the first studies to investigate the independent prospective association between activity intensity, DED and measures of adiposity in a population-based sample of young adolescents. No evidence was shown for a prospective association between SED, MPA or VPA and adiposity indicators, whereas the evidence for a prospective association between DED and adiposity was mixed and varied by outcome and analytical approach applied. On the basis of these results, no robust conclusions can be drawn on the associations of the impact of activity intensity and DED with weight gain. Future work should focus on analysing longitudinal data using diverse approaches in sufficiently large samples, with valid measures of the behaviours and sufficient follow-up. In addition, the role of reverse causality and the potential prospective impact of activity intensity and dietary behaviours on non-adiposity outcomes (such as mental well-being, academic performance and bone health) should be considered more consistently to inform public health policy. From a public health perspective, promoting increased physical activity and healthy eating, and decreased consumption of energy-dense foods, remains an important public health target, even if such changes may have minimal impact on adiposity.

\section{Acknowledgements}

The authors thank the schools, children and parents for their participation in the SPEEDY study. The authors also thank everyone who helped with the data collection process and the Norfolk Children's Services for their invaluable input and support. In addition, the authors thank Rebekah Steele, Kate Westgate and Stefanie Mayle from the physical activity technical team at the Medical Research Council (MRC) Epidemiology Unit for their assistance in processing the accelerometer data.

The SPEEDY study is funded by the National Prevention Research Initiative (http://www.npri.org.uk), consisting of the following funding partners: British Heart Foundation; Cancer Research UK; Department of Health; Diabetes UK; Economic and Social Research Council; MRC; Health and Social Care Research and Development Office for the Northern Ireland; Chief Scientist Office, Scottish Government Health Directorates; Welsh Assembly Government; and World Cancer Research Fund. This work was also supported by the MRC (Unit Programme numbers MC_UU_12015/3; MC_UU_12015/4; MC_UU_12015/7; U105960389) and the Centre for Diet and Activity Research, a UK Clinical Research Collaboration (UKCRC) Public Health Research: Centre of Excellence. Funding from the British Heart Foundation, Economic and Social Research Council, MRC, the National Institute for Health Research and the Wellcome Trust, under the auspices of the UKCRC, is gratefully acknowledged.

E. M. F. v. S., S. J. G., U. E. and A. C. designed and conducted the research; S. J. S. performed the statistical analyses; G. L. A. and A. C. led on entry and interpretation of the dietary data; E. M. F. V. S. wrote the paper with all the other authors providing critical input. All the authors take responsibility for the final content; primary responsibility lies with E. M. F. v. S.

None of the authors has any conflicts of interest to declare.

\section{References}

1. Gortmaker SL, Swinburn BA, Levy D, et al. (2011) Changing the future of obesity: science, policy, and action. Lancet $\mathbf{3 7 8}$, 838-847.

2. Health and Social Care Information Centre (2013) National Child Measurement Programme: England, 2012/13 School Year. Leeds: Health and Social Care Information Centre.

3. Ogden CL, Carroll MD, Kit BK, et al. (2014) Prevalence of childhood and adult obesity in the United States, 2011-2012. JAMA 311, 806-814.

4. Waters E, de Silva-Sanigorski A, Hall BJ, et al. (2011) Interventions for preventing obesity in children. The Cochrane Database of Systematic Reviews 2011, CD001871. http:// onlinelibrary.wiley.com/doi/10.1002/14651858.CD001871.pub3/ abstract;jsessionid=E2980F3DB1C295FDDAD8A0B84B93AA00. f03t03 
5. Summerbell CD, Douthwaite W, Whittaker V, et al. (2009) The association between diet and physical activity and subsequent excess weight gain and obesity assessed at 5 years of age or older: a systematic review of the epidemiological evidence. Int J Obes (Lond) 33 , Suppl. 3, S1-S92.

6. Tanaka C, Reilly JJ \& Huang WY (2014) Longitudinal changes in objectively measured sedentary behaviour and their relationship with adiposity in children and adolescents: systematic review and evidence appraisal. Obes Rev 15, 791-803.

7. Perez-Escamilla R, Obbagy JE, Altman JM, et al. (2012) Dietary energy density and body weight in adults and children: a systematic review. J Acad Nutr Diet 112, 671-684.

8. Wilks DC, Sharp SJ, Ekelund U, et al. (2011) Objectively measured physical activity and fat mass in children: a bias-adjusted meta-analysis of prospective studies. PLOS ONE 6, e17205.

9. Wilks DC, Besson H, Lindroos AK, et al. (2011) Objectively measured physical activity and obesity prevention in children, adolescents and adults: a systematic review of prospective studies. Obes Rev 12, e119-e129.

10. Steele RM, van Sluijs EMF, Cassidy A, et al. (2009) Targeting sedentary time or moderate- and vigorous-intensity activity: independent relations with adiposity in a population-based sample of 10-y-old British children. Am J Clin Nutr 90, 1185-1192.

11. Carson V, Rinaldi RL, Torrance B, et al. (2014) Vigorous physical activity and longitudinal associations with cardiometabolic risk factors in youth. Int J Obes (Lond) 38, 16-21.

12. Tremblay MS, LeBlanc AG, Kho ME, et al. (2011) Systematic review of sedentary behaviour and health indicators in schoolaged children and youth. Int J Behav Nutr Phys Act 8, 98.

13. Saunders TJ, Chaput JP \& Tremblay MS (2014) Sedentary behaviour as an emerging risk factor for cardiometabolic diseases in children and youth. Can J Diabetes 38, 53-61.

14. Stamatakis E, Coombs N, Tiling K, et al. (2015) Sedentary time in late childhood and cardiometabolic risk in adolescence. Pediatrics 135, e1432-e1441.

15. Marques A, Minderico C, Martins S, et al. (2015) Cross-sectional and prospective associations between moderate to vigorous physical activity and sedentary time with adiposity in children. Int J Obes (Lond) (epublication ahead of print version 25 August 2015).

16. Wilks DC, Mander AP, Jebb SA, et al. (2011) Dietary energy density and adiposity: employing bias adjustments in a metaanalysis of prospective studies. BMC Public Health 11, 48.

17. Ambrosini GL (2014) Childhood dietary patterns and later obesity: a review of the evidence. Proc Nutr Soc 73, 137-146.

18. Johnson L, Mander AP, Jones LR, et al. (2008) A prospective analysis of dietary energy density at age 5 and 7 years and fatness at 9 years among UK children. Int J Obes (Lond) 32, 586-593.

19. Johnson L, van Jaarsveld CH, Emmett PM, et al. (2009) Dietary energy density affects fat mass in early adolescence and is not modified by FTO variants. PLOS ONE $\mathbf{4}$, e4594.

20. Van Sluijs E, Skidmore P, Mwanza K, et al. (2008) Physical activity and dietary behaviour in a population-based sample of British 10-year old children: the SPEEDY study (Sport, Physical activity and Eating behaviour: Environmental Determinants in Young people). BMC Public Health $\mathbf{8}, 388$.

21. Corder K, Sharp SJ, Atkin AJ, et al. (2015) Change in objectively measured physical activity during the transition to adolescence. BrJ Sports Med 49, 730-736.

22. Cole T, Freeman J \& Preece M (1995) Body mass index reference curves for the UK, 1990. Arch Dis Child 73, 25-29.

23. Wells JC, Williams JE, Haroun D, et al. (2009) Aggregate predictions improve accuracy when calculating metabolic variables used to guide treatment. Am J Clin Nutr 89, 491-499.
24. Kriemler S, Puder J, Zahner L, et al. (2010) Estimation of percentage body fat in 6- to 13-year-old children by skinfold thickness, body mass index and waist circumference. BrJ Nutr 104, 1565-1572.

25. Deurenberg P, Weststrate JA \& Seidell JC (1991) Body mass index as a measure of body fatness: age- and sex-specific prediction formulas. BrJ Nutr 65, 105-114.

26. Pietrobelli A, Faith MS, Allison DB, et al. (1998) Body mass index as a measure of adiposity among children and adolescents: a validation study. J Pediatr 132, 204-210.

27. Foster BJ, Platt RW \& Zemel BS (2012) Development and validation of a predictive equation for lean body mass in children and adolescents. Ann Hum Biol 39, 171-182.

28. Chumlea WC, Schubert CM, Reo NV, et al. (2005) Total body water volume for white children and adolescents and anthropometric prediction equations: the Fels Longitudinal Study. Kidney Int 68, 2317-2322.

29. Haroun D, Taylor SJ, Viner RM, et al. (2010) Validation of bioelectrical impedance analysis in adolescents across different ethnic groups. Obesity (Silver Spring) 18, 1252-1259.

30. Tyrrell VJ, Richards G, Hofman P, et al. (2001) Foot-to-foot bioelectrical impedance analysis: a valuable tool for the measurement of body composition in children. Int $J$ Obes Relat Metab Disord 25, 273-278.

31. Mellits ED \& Cheek DB (1970) Estimating total body water in children on the basis of height and weight: a reevaluation of the formulas of Mellits and Cheek. Monogr Soc Res Child Dev 35, 12-26.

32. Corder K, Ekelund U, Steele RM, et al. (2008) Assessment of physical activity in youth. J Appl Physiol 105, 977-987.

33. De Vries SI, Van Hirtum HW, Bakker I, et al. (2009) Validity and reproducibility of motion sensors in youth: a systematic update. Med Sci Sports Exerc 41, 818-827.

34. Mattocks C, Ness A, Leary S, et al. (2008) Use of accelerometers in a large field-based study of children: protocols, design issues, and effects on precision. J Phys Act Health 5, S98-S111.

35. Andersen LB, Harro M, Sardinha LB, et al. (2006) Physical activity and clustered cardiovascular risk in children: a crosssectional study (The European Youth Heart Study). Lancet 368, 299-304.

36. Brage S, Brage N, Wedderkopp N, et al. (2003) Reliability and validity of the computer science and applications accelerometer in a mechanical setting. Meas Phys Educ Exerc Sci 7 , 101-119.

37. Trost S, Ward D, Moorehead S, et al. (1998) Validity of the computer and science and applications (CSA) activity monitor in children. Med Sci Sports Exerc 30, 629-633.

38. Crawford PB, Obarzanek E, Morrison J, et al. (1994) Comparative advantage of 3-day food records over 24-hour recall and 5-day food frequency validated by observation of 9- and 10-year-old girls. J Am Diet Assoc 94, 626-630.

39. Prynne CJ, Handford C, Dunn V, et al. (2013) The quality of midday meals eaten at school by adolescents; school lunches compared with packed lunches and their contribution to total energy and nutrient intakes. Public Health Nutr 16, $1118-1125$

40. Crawley H (2002) Food Portion Sizes, 3rd ed. London: The Stationery Office (formerly HMSO).

41. Howell Davies O, Suleiman S, Nicholas J, et al. (2008) Food portion weights in primary and secondary school lunches in England. J Hum Nutr Diet 21, 46-62.

42. Wrieden WL, Longbottom PJ, Adamson AJ, et al. (2008) Estimation of typical food portion sizes for children of different ages in Great Britain. Br J Nutr 99, 1344-1353. 
43. Fitt E, Cole D, Ziauddeen N, et al. (2015) DINO (Diet In Nutrients Out) - an integrated dietary assessment system. Public Health Nutr 18, 234-241.

44. Johnson L, Wilks DC, Lindroos AK, et al. (2009) Reflections from a systematic review of dietary energy density and weight gain: is the inclusion of drinks valid? Obes Rev 10, 681-692.

45. Livingstone MB, Robson PJ \& Wallace JM (2004) Issues in dietary intake assessment of children and adolescents. $\mathrm{BrJ}$ Nutr 92, Suppl. 2, S213-S222.

46. Torun B (2005) Energy requirements of children and adolescents. Public Health Nutr 8, 968-993.

47. Livingstone MB, Robson PJ, Black AE, et al. (2003) An evaluation of the sensitivity and specificity of energy expenditure measured by heart rate and the Goldberg cut-off for energy intake: basal metabolic rate for identifying mis-reporting of energy intake by adults and children: a retrospective analysis. Eur J Clin Nutr 57, 455-463.

48. Ambrosini GL, Emmett PM, Northstone K, et al. (2012) Identification of a dietary pattern prospectively associated with increased adiposity during childhood and adolescence. Int J Obes (Lond) 36, 1299-1305.

49. Chen X, Beydoun MA \& Wang Y (2008) Is sleep duration associated with childhood obesity? A systematic review and meta-analysis. Obesity (Silver Spring) 16, 265-274.

50. Frisancho AR (2000) Prenatal compared with parental origins of adolescent fatness. Am J Clin Nutr 72, 1186-1190.
51. Reilly JJ, Armstrong J, Dorosty AR, et al. (2005) Early life risk factors for obesity in childhood: cohort study. BMJ 330, 1357.

52. Wareham NJ, van Sluijs EM \& Ekelund U (2005) Physical activity and obesity prevention: a review of the current evidence. Proc Nutr Soc 64, 229-247.

53. Chinapaw MJ, Proper KI, Brug J, et al. (2011) Relationship between young peoples' sedentary behaviour and biomedical health indicators: a systematic review of prospective studies. Obes Rev 12, e621-e632.

54. Hjorth MF, Chaput JP, Ritz C, et al. (2014) Fatness predicts decreased physical activity and increased sedentary time, but not vice versa: support from a longitudinal study in 8- to 11-year-old children. Int J Obes (Lond) 38, 959-965.

55. Ekelund U, Luan J, Sherar LB, et al. (2012) Moderate to vigorous physical activity and sedentary time and cardiometabolic risk factors in children and adolescents. JAMA 307, 704-712.

56. Ekelund U, Brage S, Besson H, et al. (2008) Time spent being sedentary and weight gain in healthy adults: reverse or bidirectional causality? Am J Clin Nutr 88, 612-617.

57. Golubic R, Wijndaele K, Sharp SJ, et al. (2015) Physical activity, sedentary time and gain in overall and central body fat: 7-year follow-up of the ProActive trial cohort. Int J Obes (Lond) 39, 142-148.

58. Hutcheon JA, Chiolero A \& Hanley JA (2010) Random measurement error and regression dilution bias. BMJ 340, c2289. 